\title{
Effect of the Interfacial Adhesion on the Tensile and Impact Properties of Carbon Fiber Reinforced Polypropylene Matrices
}

\author{
Clara Leal Nogueira ${ }^{\mathrm{a}, \mathrm{b} *}$, Jane Maria Faulstich de Paiva ${ }^{\mathrm{b} *}$, Mirabel Cerqueira Rezende ${ }^{\mathrm{b} *}$ \\ a Departamento de Engenharia Aeronáutica e Mecânica / ITA \\ Praça Marechal-do-Ar Eduardo Gomes, 50, 12228-904 São José dos Campos - SP, Brazil \\ ${ }^{\mathrm{b}}$ Divisão de Materiais- AMR / IAE / CTA - Centro Técnico Aeroespacial \\ Praça Marechal-do-Ar Eduardo Gomes, 50, 12228-904 São José dos Campos - SP, Brazil
}

Received: October 29, 2003; Revised: November 22, 2004

\begin{abstract}
Thermoplastic composites have been applied in a wide variety of industrial products, showing recently a great potential to be used in aeronautical field. The objectives of this work were to evaluate the fiber/matrix interface of carbon fiber reinforced polypropylene-based matrices after tensile and impact tests and also to compare the mechanical test results of the manufactured laminates. The laminates were prepared by stacking carbon fiber fabric style Plain Weave (CF) and films of four different polypropylene matrices, described as (a) polypropylene-PP, (b) polypropylene-polyethylene copolymer-PP-PE, (c) PP-PE with an interfacial compatibilizer-AM1 and (d) PP-PE containing an elastomeric modifier-AM2. The composites were processed using hot compression molding. The mechanical testing results showed that the CF-AM1 laminate family presented the lowest impact strength and the highest tensile strength values when compared to the other laminates. SEM analysis observations of both tensile and impact fractured specimens of the CF-PP/PE-AM1 specimens revealed a stronger fiber/matrix interface. The CF-PP/PE-AM2 laminate showed a lower tensile strength and higher impact strength values when compared to the CF-PP/PE-AM1 one. PP-PE and PP laminates presented the lowest impact strength values.
\end{abstract}

Keywords: polypropylene, composites, carbon fiber, mechanical properties

\section{Introduction}

Polypropylene and polyethylene matrices are very used to obtain several components for automotive industry because of their low cost, low density and the possibility to obtain recyclable materials ${ }^{1}$. An application few explored for these polymers is their utilization in aeronautical field for obtaining continuous fiber reinforced thermoplastic composites ${ }^{2}$, aiming for structural components that are not submitted to wide range of temperatures.

Thermoplastic composite systems can offer some advantages over composites based on thermosetting resins. Among them, it can be cited the absence of both time for consuming and chemical reactions during the processing, resulting in a relatively fast production of components with complex shapes and also the possibility of transforming a shape in another ${ }^{3-5}$. Other factors that become the thermoplastics attractive are: a simpler storage and easy handling of materials, indefinite shelf life without refrigeration, fewer repairs because of the higher toughness and more ductility than the thermoset ones ${ }^{6-9}$.

Aeronautical applications require improvements in mechanical properties, as for example, impact strength, because the components are frequently exposed to several types of impact. Accidental impacts of various natures can be caused by dropped tools, accidental crashing and other impacts occurred during the manufacturing processes and also in service ${ }^{10}$.

An option to improve the damage resistance is by using carbon fiber as reinforcement. This possibility has motivated studies involving the susceptibility to impact damages of the reinforced polymer composites.

In view of the opportunity, the present work shows the manufacture of composites based on polypropylene (PP) and copolymer (PP/ $\mathrm{PE})$ matrices reinforced with carbon fabric, evaluating the increase of impact energy absorbing capability of these thermoplastic composites.

*e-mail: clara@iae.cta.br, janepaiva@uol.com.br, mirabel@iae.cta.br

Article presented at the II SBPMat, Rio de Janeiro - RJ, 26-29/October/2003
This study uses modified thermoplastic polymer matrices looking for improvements of the carbon fiber/matrix interface of the manufactured composites. The performance of the manufactured thermoplastic composites was evaluated by tensile and impact tests.

The process used to obtain the laminate composites was based on alternate stacking of carbon fiber fabric and thermoplastic films instead of thermoplastic pre-impregnated (prepreg) lamination. This process is believed to be at least 60 percent less expensive than the prepreg conventional processing, which it is very used for the thermosetting composite manufacture.

\section{Experimental}

\subsection{Composite molding}

The reinforced thermoplastic composites were processed by hot compression molding, by stacking alternately a layer of carbon fabric style Plain Weave and two layers of thermoplastic film. This step used fifteen layers of carbon fabric, resulting in a final laminate with approximately $3 \mathrm{~mm}$ of thickness. The processed laminate, according to the literature ${ }^{11}$, is called cross ply composite, i.e., it presents the reinforcement orientation of $0 / 90^{\circ}$.

Four different thermoplastic polypropylene matrices were used. These polymers were supplied in films with thickness of $3.5 \mu \mathrm{m}$ by Polibrasil Resinas S/A, described as (a) polypropylene-PP, (b) polypropylene-polyethylene copolymer-PP-PE, (c) PP-PE containing an interfacial compatibilizer-AM1 and (d) PP-PE modified with an elastomeric modifier-AM2. The two latter thermoplastic films were developed specially for this study.

Thermoplastic polymer plates (without reinforcement) were also 
molded using the same equipment and the same molding parameters those ones used for the processed reinforced composite laminates. The thermoplastic plates and the reinforced laminates were obtained at heating rate of $5{ }^{\circ} \mathrm{C} / \mathrm{min}$ from room temperature until $230^{\circ} \mathrm{C}$. At this maximum temperature it was applied a pressure of $4 \mathrm{MPa}$ for two hours. Afterwards, the laminates were cooled until the room temperature inside the mold disposed in a hydraulic press.

After molding, the homogeneity of the carbon fiber distribution in the laminates was evaluated by microscopic analyses and the fiber volume contents were determined according to ASTM-D3171. In this method, the fiber content is measured by matrix acid digestion of a weighed composite specimen. The residue (carbon fiber) is filtered, washed, dried and weighed. This procedure was made for ten specimens of each laminate family.

\subsection{Tensile tests}

The tensile tests were carried out in accordance to ASTM D3039, using ten specimens for each family of carbon fiber reinforced composite. For the tests, it was used a universal testing machine at a constant cross-speed of $2 \mathrm{~mm} / \mathrm{min}$. The modulus of elasticity was obtained using strain gauges. The tensile strengths were calculated in accordance to ASTM D3039.

\subsection{Impact tests}

For the impact tests, specimens were cut from the laminates attending the dimensions of $100 \mathrm{~mm} \times 100 \mathrm{~mm}$ (length and width). For this step it was used a diamond saw.

The impact tests were performed using an instrumented impact machine, assisted with a falling dart, based on ASTM 5628. The impact energy, the displacement and the energy absorbed by specimens were registered.

The impact tests of the PP and PP/PE plates were carried out at room temperature using a dart of $5.3 \mathrm{~kg}$ dropped from $30 \mathrm{~cm}$ of height. For the tests, a hydraulic device of the impact equipment clamped the specimens. The used dart presents a hemispherical steel tip of diameter of $12.7 \mathrm{~mm}$.

The impact tests of the carbon fiber reinforced laminates were carried out at room temperature using darts with different weights
(5.3 kg, $12.1 \mathrm{~kg}$ and $22.9 \mathrm{~kg}$ ), but with the same tip used for the tests of the polymer plates. The specimens were hit with the darts dropped from $30 \mathrm{~cm}, 60 \mathrm{~cm}$ and $90 \mathrm{~cm}$ of height. Table 1 summarizes the conditions used for the impact tests. For each test condition and material type it was used six specimens. Hence, it was tested a total of one hundred and two specimens.

\subsection{SEM observations}

After the impact and tensile tests the fractured specimens were photographed in details and analyzed by Scanning Electron Microscopy (SEM). For these analyses it was not necessary any special preparation of the samples because they present certain conductivity due to the carbon fibers. Moreover, the equipment used has a device of variable press ( $\mathrm{Vpi}$ ).

\section{Results and Discussion}

\subsection{Carbon fiber content}

Table 2 shows the carbon fiber content values determined according to ASTM-D3171. Comparing the results of Table 2, it is observed a good agreement between the calculated and the determined values. The fiber volume contents (nearly $60 \%$ ) agree with the literature values $^{12,13}$. These results suggest that the used processing parameters are adequate, promoting a repetitive and a reliable molding process. This observation is confirmed by SEM micrographs (Figure 1), which show that the four thermoplastic laminate families present a homogeneous distribution of fibers, few regions rich in matrix (polymer-PP or copolymer PP/PE), the absence of damages in the carbon reinforcement, and no presence of voids.

The literature ${ }^{12,13}$ reports that the continuous carbon fiber reinforced thermoplastic composites can present fiber volumes varying between 60 to $70 \%$. These materials have been introduced as structural composite materials for high performance aeronautical applications ${ }^{12,13}$.

\subsection{Impact tests}

To facilitate the discussion involving the impact test results it

Table 1. Conditions of the impact tests carried out.

\begin{tabular}{lcccc}
\hline \multicolumn{1}{c}{ Code } & $\begin{array}{c}\text { Weight of } \\
\text { dart }(\mathrm{kg})\end{array}$ & Drop height $(\mathrm{cm})$ & $\begin{array}{c}\text { Impact velocity } \\
(\mathrm{m} / \mathrm{s})\end{array}$ & Impact energy $(\mathrm{J})$ \\
\hline CF-PP & 5.3 & 30 & 2.48 & 3.47 \\
CF-PP & 5.3 & 60 & 3.96 & $30.24 \pm 0.04$ \\
CF-PP & 5.3 & 90 & 3.91 & $39.51 \pm 0.05$ \\
CF-PP & 12.1 & 90 & 2.48 & $92.61 \pm 0.03$ \\
CF-PP/PE & 5.3 & 30 & 3.47 & $15.43 \pm 0.03$ \\
CF-PP/PE & 5.3 & 60 & 3.96 & $30.27 \pm 0.01$ \\
CF-PP/PE & 5.3 & 90 & 3.91 & $39.53 \pm 0.01$ \\
CF-PP/PE & 12.1 & 90 & 3.41 & $92.61 \pm 0.02$ \\
CF-PP/PE & 22.9 & 60 & 3.47 & $132.80 \pm 0.24$ \\
CF-PP/PE (AM1) & 5.3 & 60 & 3.96 & $30.25 \pm 0.03$ \\
CF-PP/PE (AM1) & 5.3 & 90 & 3.91 & $39.61 \pm 0.06$ \\
CF-PP/PE (AM1) & 12.1 & 90 & 2.48 & $92.62 \pm 0.02$ \\
CF-PP/PE (AM2) & 5.3 & 30 & 3.47 & $15.47 \pm 0.02$ \\
CF-PP/PE (AM2) & 5.3 & 60 & 3.96 & $30.28 \pm 0.03$ \\
CF-PP/PE (AM2) & 5.3 & 90 & 3.91 & $39.56 \pm 0.05$ \\
CF-PP/PE (AM2) & 12.1 & 90 & 3.41 & $92.62 \pm 0.03$ \\
CF-PP/PE (AM2) & 22.9 & 60 & & $133.00 \pm 0.14$ \\
\hline
\end{tabular}


is useful to mention that the impact is classified according to the energy level as low and high. If specimens are damaged, but without complete fracture, this situation characterizes low energy impact. If specimens are totally fractured, with the complete penetration of the dart, characterizes high-energy impact ${ }^{14}$ and the impact strength can be calculated.

Table 3 shows the impact test results for the PP polymer and $\mathrm{PP} / \mathrm{PE}$ copolymer plates as well for the carbon fiber reinforced laminates. In this way, the impact tests were carried out beginning with low energy impacts, which were continuously increased until the total fracture of the specimens.

It was observed that the polymer plates without reinforcement presented total fracture with impacts of low energy (approximately $15 \mathrm{~J})$. This process is verified by the full penetration of the dart into the material. In this case, the dart passes through the specimen and the energy involved in the impact is consumed by both the fracture process and the specimen, as vibrational energy within the sample ${ }^{15}$.

Table 3 shows a significant increase of the impact energy absorbed by the carbon fiber reinforced thermoplastic laminates when compared to the polymer or copolymer plates (material without reinforcement). Figure 2 shows in macroscopic scale the fragile behavior of the polymeric plates (Figures 2a-2b) in relation to the reinforced laminates (Figures 2c-2d). In this case, it is observed the deformation of the laminates as a consequence of the favorable combination of carbon fiber reinforcement with the thermoplastic matrix, which presents higher capability to deform without fracture.

Tables 3 and 4 present the types of damages occurred in the tested laminates. The observation named small damage describes the beginning of delamination; medium is related to the delamination of some layers; partially fractured describes the delamination of many layers

Table 2. Carbon fiber contents of the composites (laminates) manufactured.

\begin{tabular}{lccc}
\hline \multicolumn{1}{c}{ Code } & $\begin{array}{c}\text { Weight }(\%) \\
\text { (calculated) }\end{array}$ & $\begin{array}{c}\text { Weight }(\%) \\
\text { (determined according to } \\
\text { ASTMD3171) }\end{array}$ & CF Volume (\%) \\
\hline CF-PP & $74.7 \pm 0.3$ & $75.0 \pm 0.3$ & $60.2 \pm 0.4$ \\
CF-PP/PE & $74.9 \pm 0.3$ & $75.0 \pm 0.4$ & $60.4 \pm 0.4$ \\
CF-PP/PE-AM1 & $74.4 \pm 0.5$ & $74.9 \pm 0.8$ & $59.8 \pm 0.6$ \\
CF-PP/PE-AM2 & $74.7 \pm 0.3$ & $74.8 \pm 0.3$ & $60.3 \pm 0.4$ \\
\hline
\end{tabular}

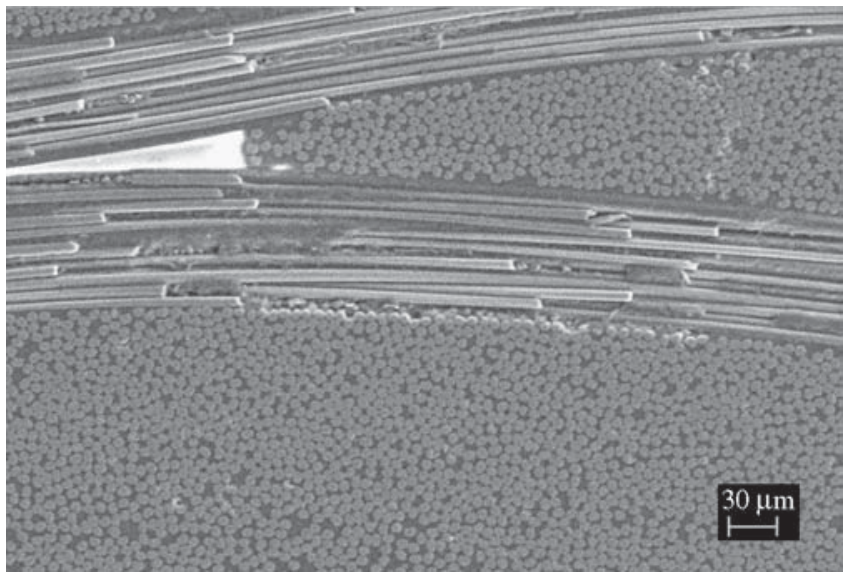

CF-PP (200 x)

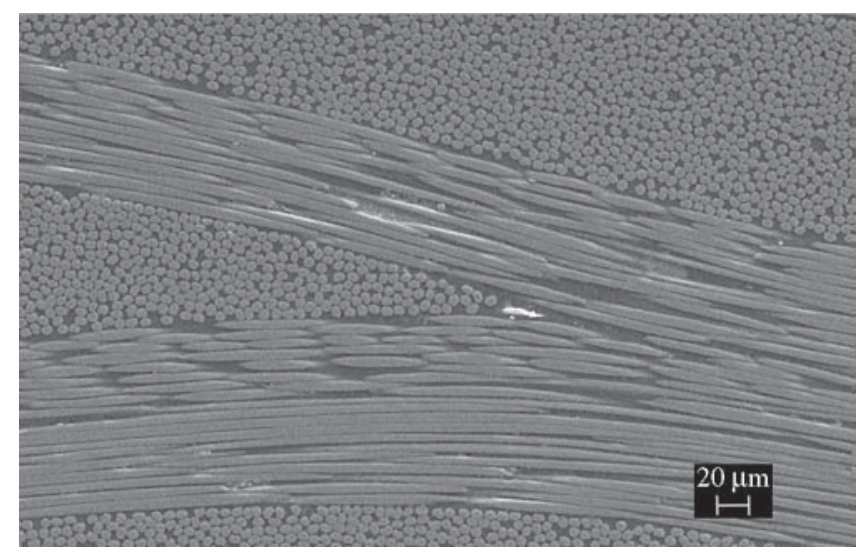

CF-PP/PE-AM1 (200 x)

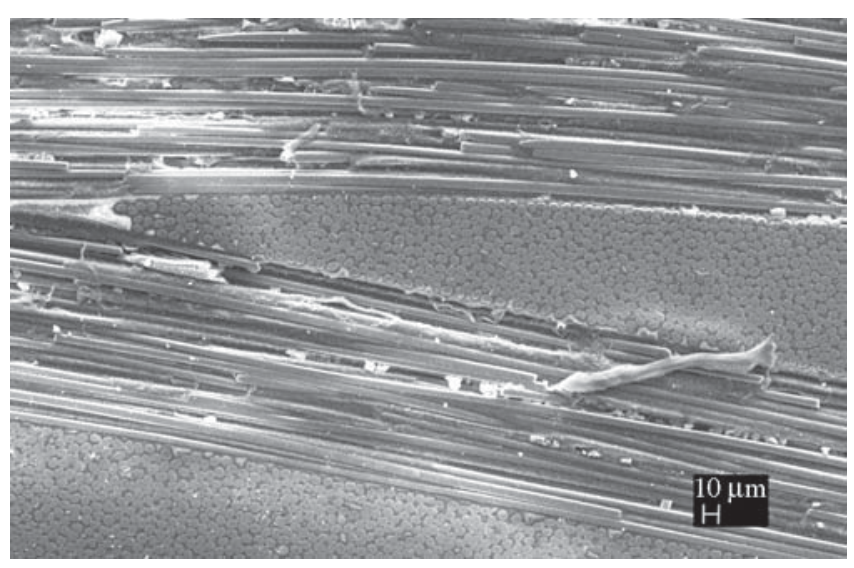

CF-PP/PE (200 x)

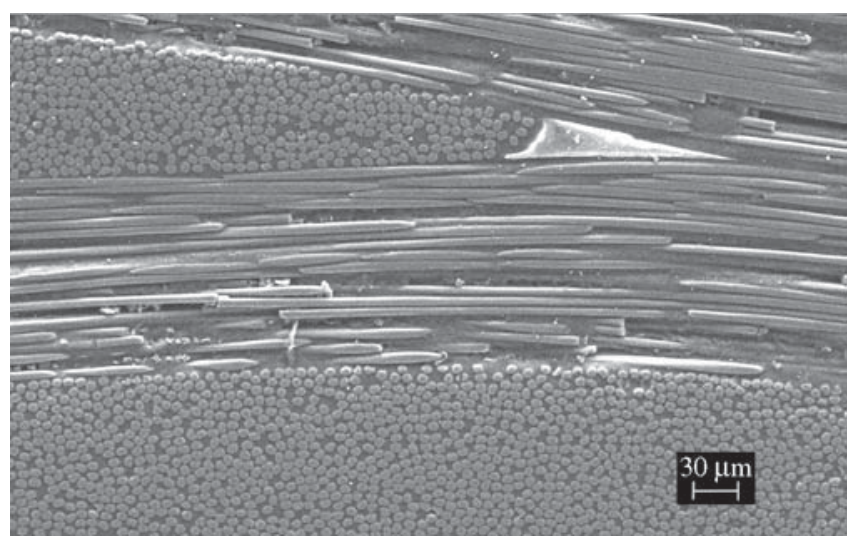

CF-PP/PE-AM2 (200 x)

Figure 1. SEM of the laminates molded. 
Table 3. Impact results of the laminates manufactured with thermoplastic matrix.

\begin{tabular}{|c|c|c|c|c|}
\hline Code & Impact Energy $(\mathrm{J})$ & $\begin{array}{l}\text { Total Absorbed Energy } \\
\text { (J) }\end{array}$ & Damage & $\begin{array}{l}\text { Impact Strength } \\
(\mathrm{J} / \mathrm{cm})\end{array}$ \\
\hline $\mathrm{PP}$ & $14.58 \pm 0.03$ & $1.30 \pm 0.35$ & fractured & 6.3 \\
\hline PP/PE copolymer & $16.07 \pm 0.04$ & $8.96 \pm 4.15$ & fractured & 41.3 \\
\hline CF-PP & $15.48 \pm 0.04$ & $14.72 \pm 0.11$ & small & non-calculated \\
\hline CF-PP & $30.24 \pm 0.04$ & $29.07 \pm 0.07$ & medium & non-calculated \\
\hline CF-PP & $39.51 \pm 0.05$ & $38.91 \pm 0.14$ & partially fractured & $139.1 \pm 2.6$ \\
\hline CF-PP & $92.61 \pm 0.03$ & $37.51 \pm 4.51$ & fractured & $133.4 \pm 17.4$ \\
\hline CF-PP/PE & $15.43 \pm 0.03$ & $14.39 \pm 0.06$ & small & non-calculated \\
\hline CF-PP/PE & $30.27 \pm 0.01$ & $29.39 \pm 0.12$ & small & non-calculated \\
\hline CF-PP/PE & $39.53 \pm 0.01$ & $38.97 \pm 0.22$ & medium & non-calculated \\
\hline CF-PP/PE & $92.61 \pm 0.02$ & $59.26 \pm 18.94$ & partially fractured & $213.0 \pm 71.4$ \\
\hline CF-PP/PE & $132.79 \pm 0.24$ & $34.68 \pm 4.07$ & fractured & $120.1 \pm 12.8$ \\
\hline
\end{tabular}

CF: carbon fiber fabric reinforcement

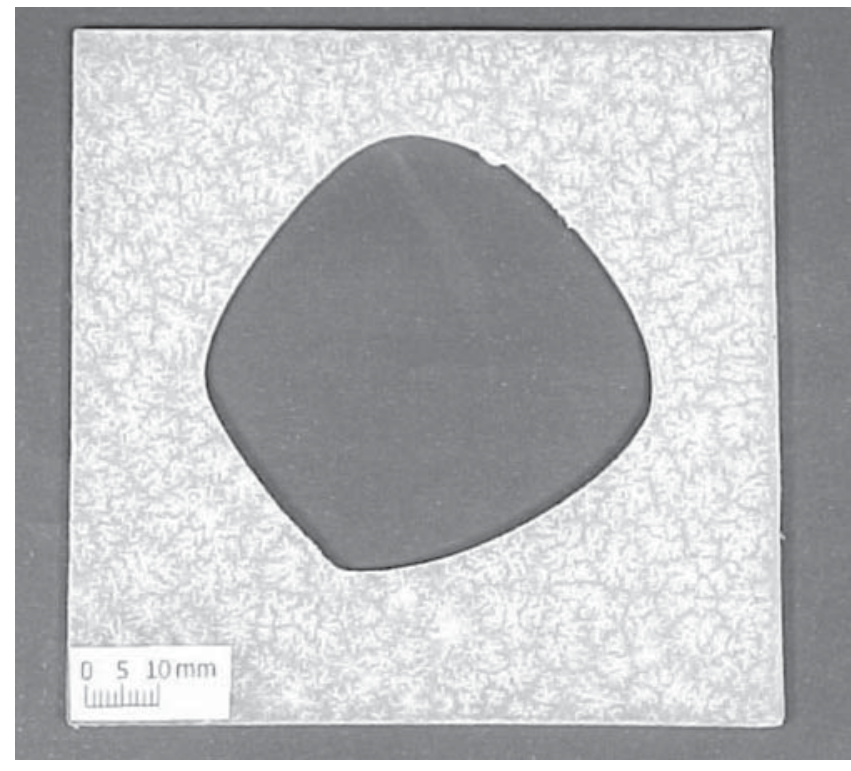

(a) Polypropylene (PP) polymer

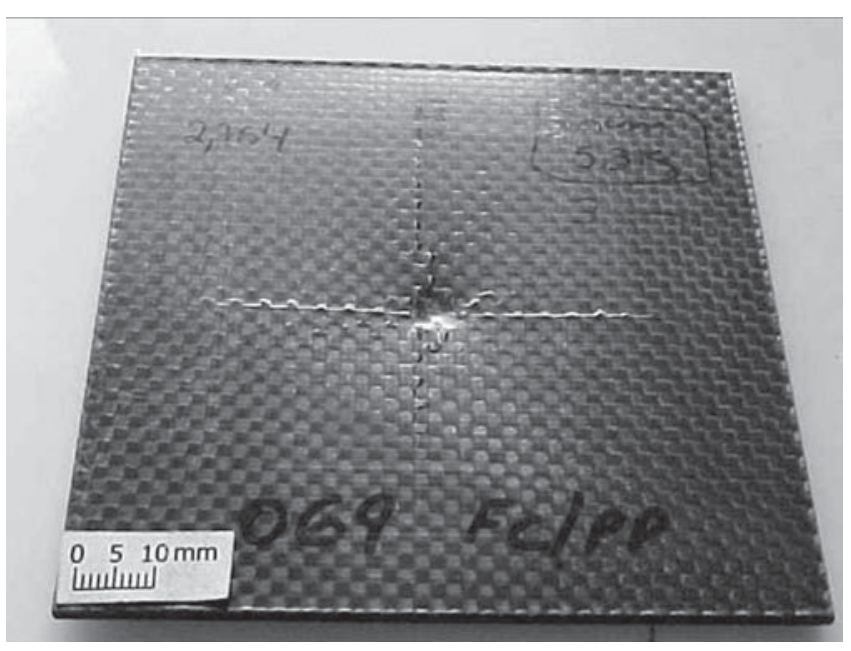

(c) CF-PP laminate

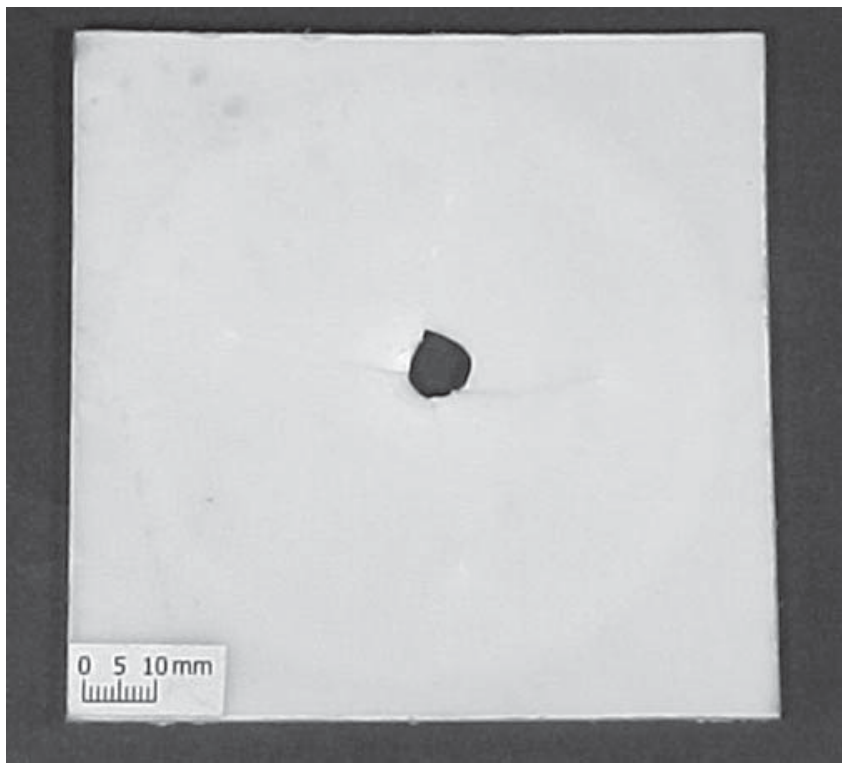

(b) Polypropylene/polyethylene (PP/PE) copolymer

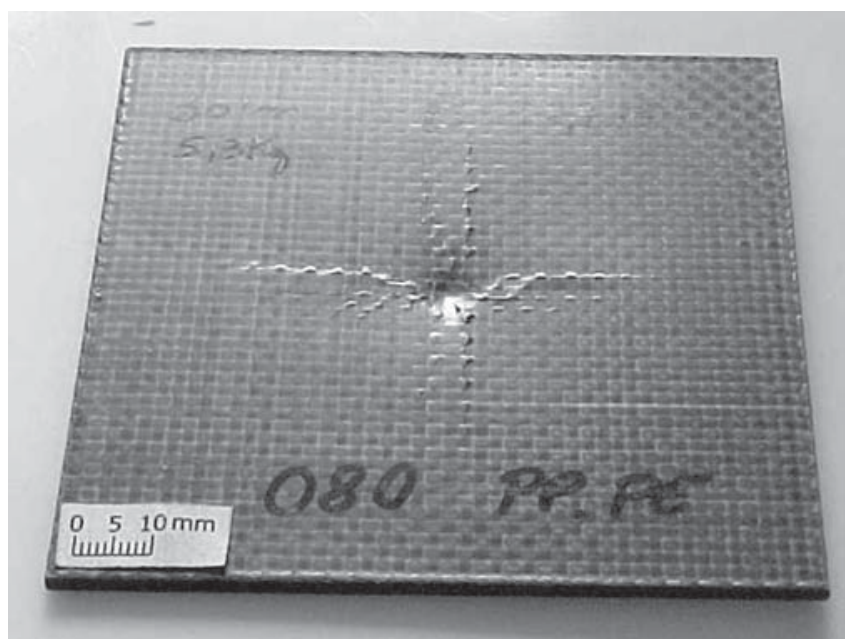

(d) CF-PP/PE laminate

Figure 2. Photographs of the specimens after the impact tests with approximately $15 \mathrm{~J}$ of energy. 
but without complete fracture and fractured when the specimen is completely penetrated by the dart. In the latter case, the specimens were impacted with sufficient energy to ensure the complete failure. This event is also called penetration resistance, which measures the total energy absorbing capability ${ }^{14,15}$. The energy value immediately before the total penetration is related to the maximum load that the material can tolerate before its complete fail. In literature this point is defined as saturation impact energy, which it is the maximum energy bearable by the material without perforation ${ }^{16,17}$.

Figures 3, 4 and 5 show the evolution of fracture of CF-PP/PE laminate according to the increase of the impact energy cited in Table 3. Figure 3 shows the beginning of delamination with the deformation of the laminate impacted with nearly $40 \mathrm{~J}$. The opposite side (back area) of the impacted samples reveals more clearly this phenomenon. Figure 4 is representative of the impact with nearly $92 \mathrm{~J}$ of energy, showing the presence of delamination in both sides of the specimen, the impacted surface and the opposite side. Already in Figure 5 is observed the total fracture caused by penetration of the dart.

Table 4 shows that the utilization of AM1 modifier improved the interfacial adhesion between fiber and matrix. This behavior is followed by a decrease of the impact strength when compared with both CF-PP/PE-AM2 and CF-PP/PE laminates without modifier (Table 3). Therefore, in accordance with the literature ${ }^{18}$, stronger interface bond not permits adequate crack diverting. This characteristic makes that the composite presents brittle behavior. A weaker interfacial bond in general leads to intraply splitting and interply delamination, allowing that the composite absorbs a larger amount of impact energy, showing that the splitting and delamination are the dominant failure modes. Meanwhile, if the interface bond is too weak, the material will not support loads in shear or compression ${ }^{18}$. Therefore, it is necessary to find a medium point as shown for the CF-PP/PE-AM2 laminate.

In accordance with the literature ${ }^{17}$, the impact damage mechanisms in laminate composites constitute a very complex process. Many times it is a combination of matrix cracking, surface buckling, delamination, fiber shear-out, and fiber fracture, which usually all interact with each other ${ }^{17}$.

In relation to the CF-PP/PE-AM2 laminate (Table 4) it can be observed higher capability to resist to damages than the other composites because the utilization of AM2 modifier increased the impact energy to fracture the composite. In this case, the used elastomer called AM2 really acted as toughening agent and improved the penetration impact resistance of the composites. As recorded in literature ${ }^{14}$, the deformation of thermoplastic is believed to be the key factor that improves the penetration impact resistance of the composites.

Figures 6 and 7 show the types of fractures occurred in the samples when fractured with higher energy, $92 \mathrm{~J}$ and $132 \mathrm{~J}$ respectively. These figures show a complete delamination in the center of the tested specimen caused by the total penetration of the dart, causing the complete failure of the material. Consequently, the specimens show a significant quantity of fiber breakage. Still, Figure 7 shows

Table 4. Impact results of the laminates manufactured with modified thermoplastic matrix.

\begin{tabular}{|c|c|c|c|c|}
\hline Code & Impact Energy $(\mathrm{J})$ & Total Absorbed Energy (J) & Damage & $\begin{array}{c}\text { Impact Strength } \\
(\mathrm{J} / \mathrm{cm})\end{array}$ \\
\hline CF-PP/PE (AM1) & $30.25 \pm 0.03$ & $30.23 \pm 0.28$ & medium & non-calculated \\
\hline CF-PP/PE (AM1) & $39.61 \pm 0.06$ & $30.36 \pm 1.12$ & partially fractured & $109.5 \pm 4.2$ \\
\hline CF-PP/PE (AM1) & $92.62 \pm 0.02$ & $30.06 \pm 1.54$ & fractured & $107.5 \pm 4.2$ \\
\hline CF-PP/PE (AM2) & $15.47 \pm 0.02$ & $14.66 \pm 0.07$ & small & non-calculated \\
\hline CF-PP/PE (AM2) & $30.28 \pm 0.03$ & $29.35 \pm 0.07$ & small & non-calculated \\
\hline CF-PP/PE (AM2) & $39.56 \pm 0.05$ & $38.86 \pm 0.13$ & medium & non-calculated \\
\hline CF-PP/PE (AM2) & $92.62 \pm 0.03$ & $85.27 \pm 17.14$ & partially fractured & $308.0 \pm 66.1$ \\
\hline CF-PP/PE (AM2) & $133.01 \pm 0.14$ & $34.80 \pm 3.68$ & fractured & $120.1 \pm 13.4$ \\
\hline
\end{tabular}

$\mathrm{CF}$ : carbon fiber fabric reinforcement

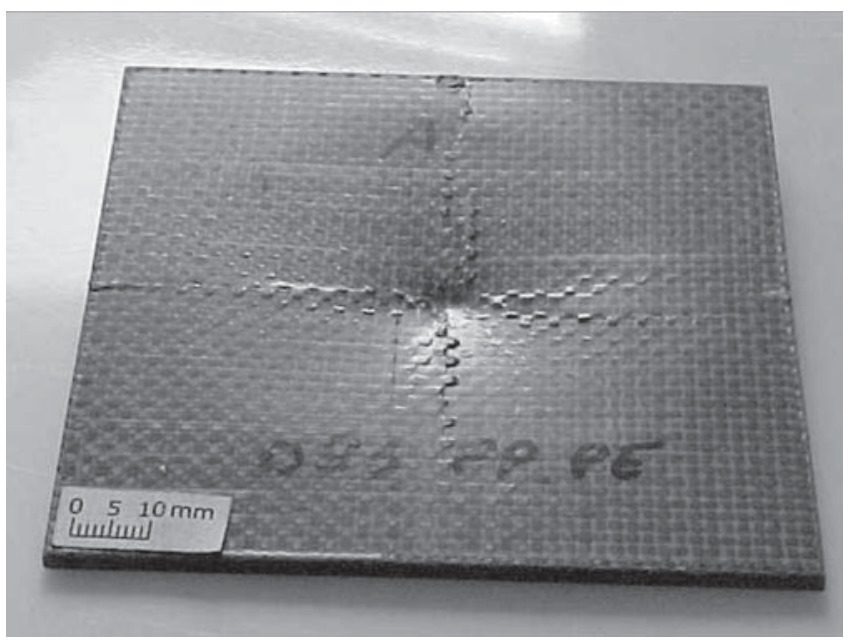

(a) CF-PP/PE laminate (front side)

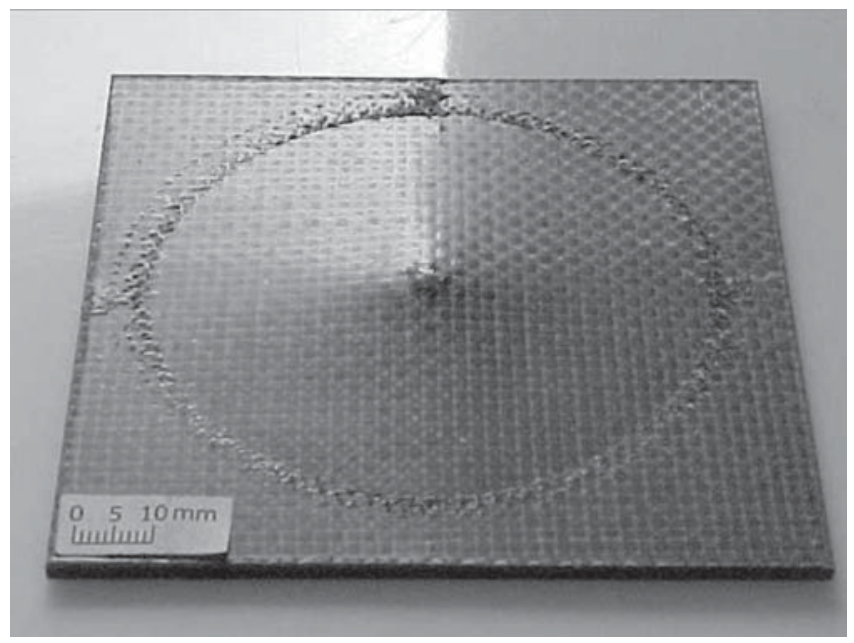

(b) CF-PP/PE laminate (opposite side)

Figure 3. Aspects of specimens after impact tests of approximately $40 \mathrm{~J}$ of energy. 


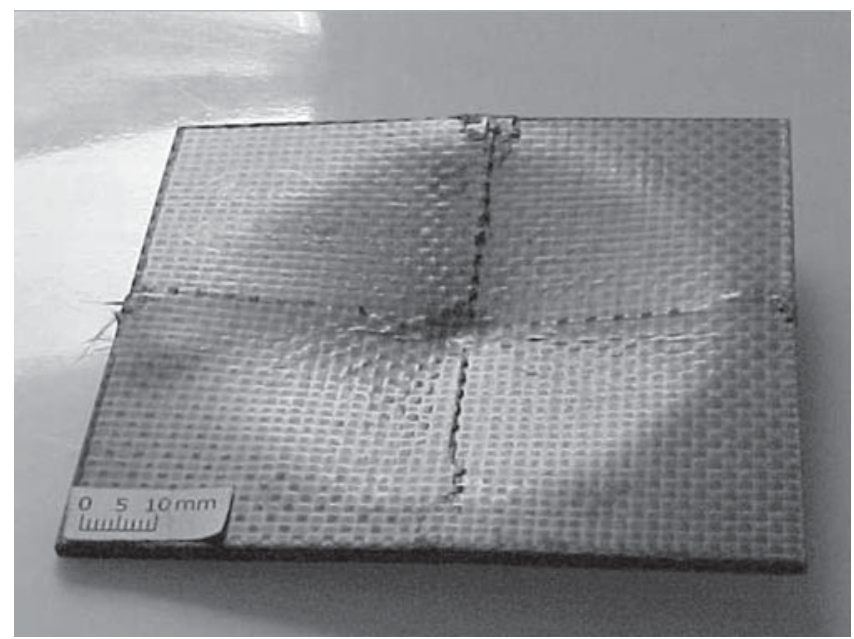

(a) CF-PP/PE laminate (front side)

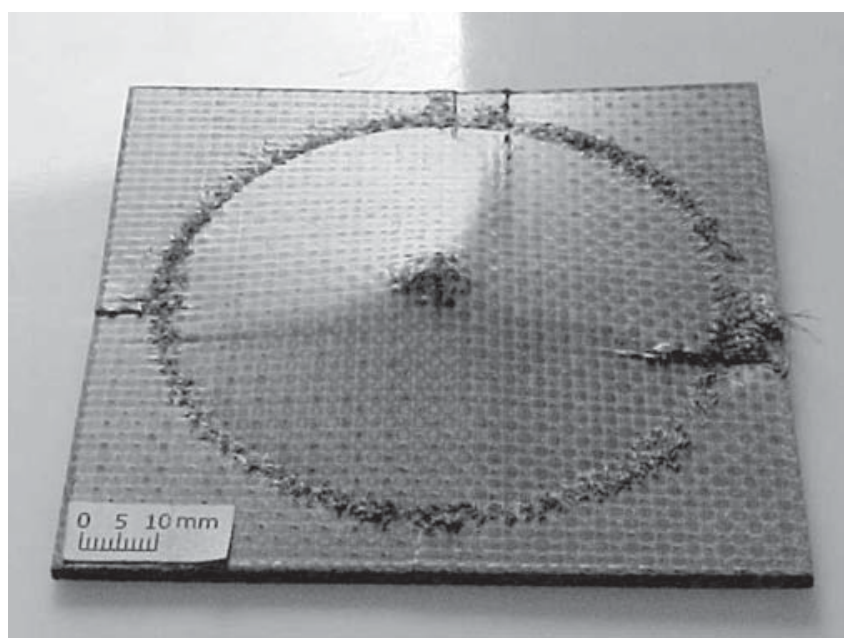

(b) CF-PP/PE laminate (opposite side)

Figure 4. Aspects of specimens after impact tests of approximately $92 \mathrm{~J}$ of energy.

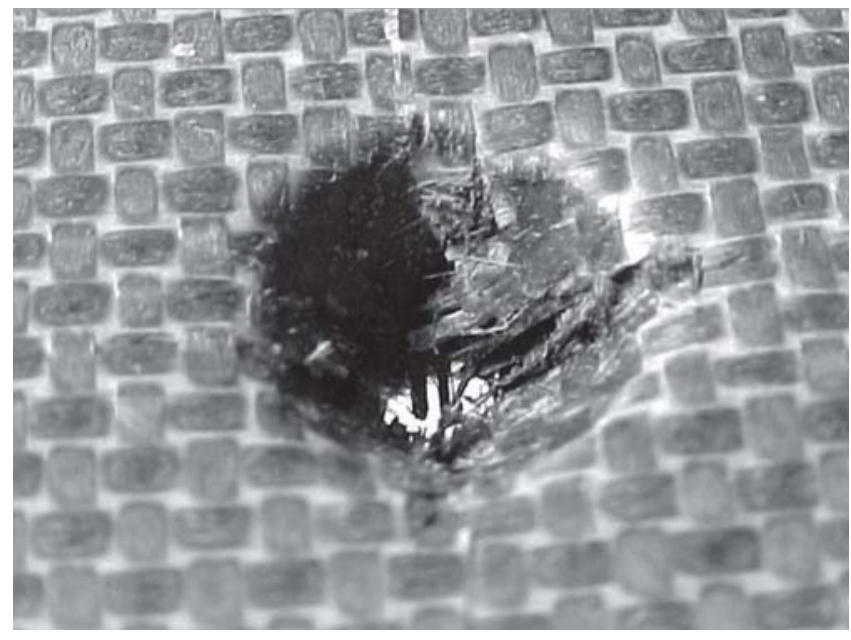

(a) CF-PP/PE laminate (front side)

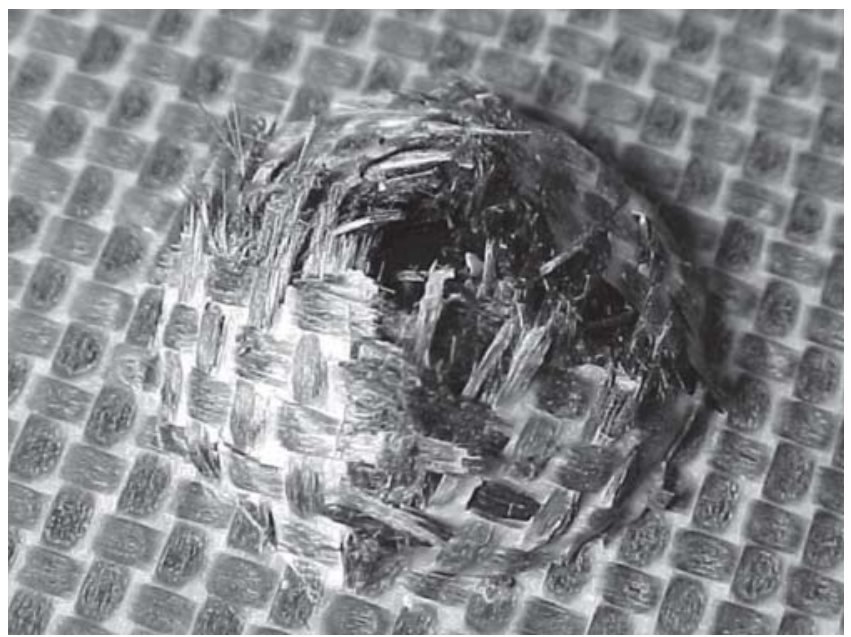

(b) CF-PP/PE laminate (opposite side)

Figure 5. Aspects of specimens after impact tests of approximately $132 \mathrm{~J}$ of energy.

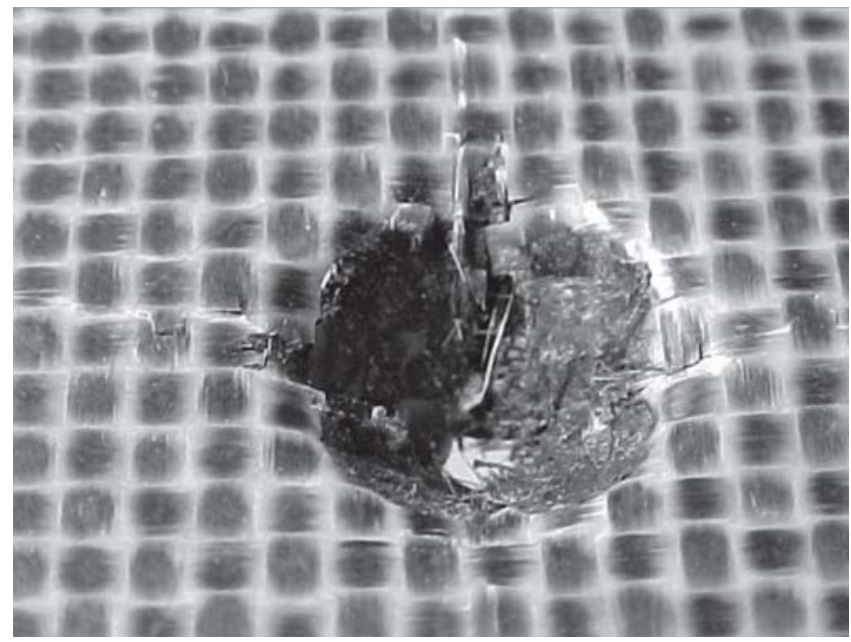

(a) CF-PP/PE (AM1) laminate (front side)

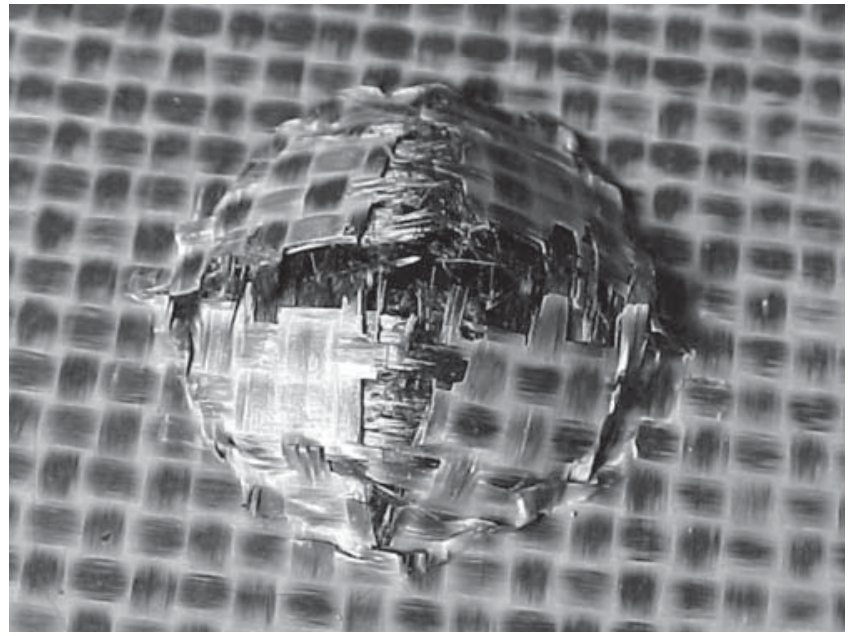

(b) CF-PP/PE (AM1) laminate (opposite side)

Figure 6. Aspects of the CF-PP/PE (AM1) specimens after impact tests with approximately $92 \mathrm{~J}$ of energy. 
that the thermoplastic matrix modified with elastomeric phase (AM2) deformed more plastically prior to fracture, when compared to the other non-modified matrices, in despite of the utilization of higher energy impact (132 J). These results agree with the literature ${ }^{14}$.

\subsection{SEM analysis}

In accordance with SEM analysis it is observed that the thermoplastic matrices without modifiers exhibit a poor recovering property of the fibers, showing consequently a weak interfacial adhesion (Figures $8 \mathrm{a}-8 \mathrm{~b}$ ). When it is used a coupling agent (AM1), the fibers show a more homogeneous recovering by the matrix (Figure 9a), resulting in a higher interfacial adhesion. In Figure $9 \mathrm{~b}$ it is observed elastomeric phases in the interfacial region of fibers-matrix, which contributed for a higher plastic deformation of this thermoplastic matrix when compared to the non-modified ones (Figure 8).

\subsection{Tensile tests}

Table 5 shows the tensile results of the manufactured laminates. Comparing the results it can be seen that CF-PP/PE-AM1 showed the highest tensile strength value, confirming the influence of the coupling modifier
(AM1), which improves the fiber-matrix interface in the composite.

In tensile tests, the filaments of the carbon fiber tow in a laminate fail at different stress levels as the applied tensile load increases. It is known from literature some main failure modes in tensile ${ }^{19,20}$. In the present work, the laminates with non-modified matrices (CF-PP and CF-PP/PE) presented the lowest tensile strength values and the main failure mechanism occurred as fiber-matrix debonding. In this case, cracks at different cross sections of the laminate joined together causing fiber-matrix debonding or shear failure of the matrix. These types of matrix shear failures and fiber-matrix debonding occurred either independently or in combination; i. e., portions of the failure path exhibited debonding, while matrix shear failure was evident in other regions. This behavior is described in literature ${ }^{20}$.

Laminates containing coupling agent in the matrix, as CF-PP/PEAM1, revealed simultaneously the breakage of fibers and matrix, evidencing better interfacial adhesion, leading to higher tensile strength and smaller impact strength values than the other laminates. The CF-PP/PE-AM2 laminate showed the opposite effect, i.e., lower tensile strength results than the other laminates, including the CF-PP laminate. The CF-PP/PE-AM2 laminate behavior is related to higher

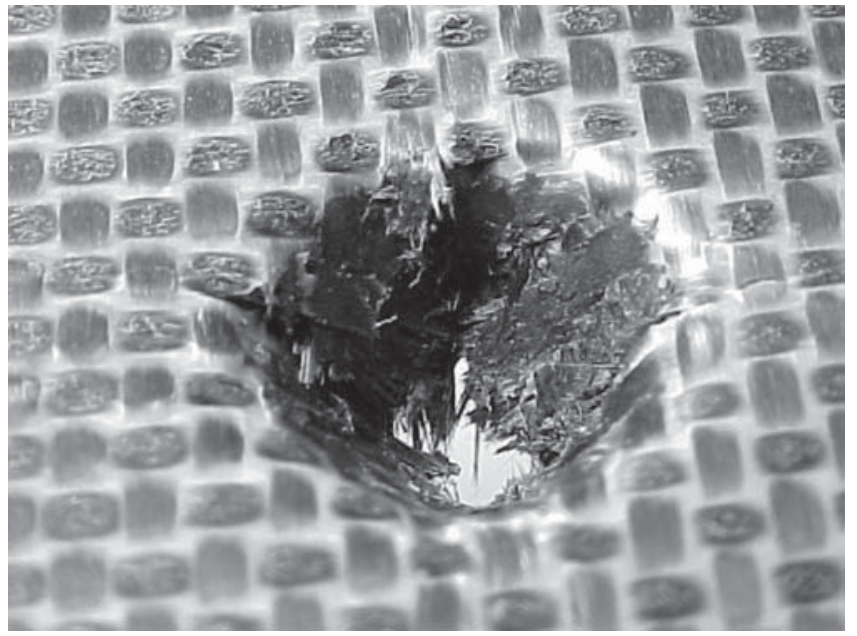

(a) CF-PP/PE (AM2) laminate (front side)

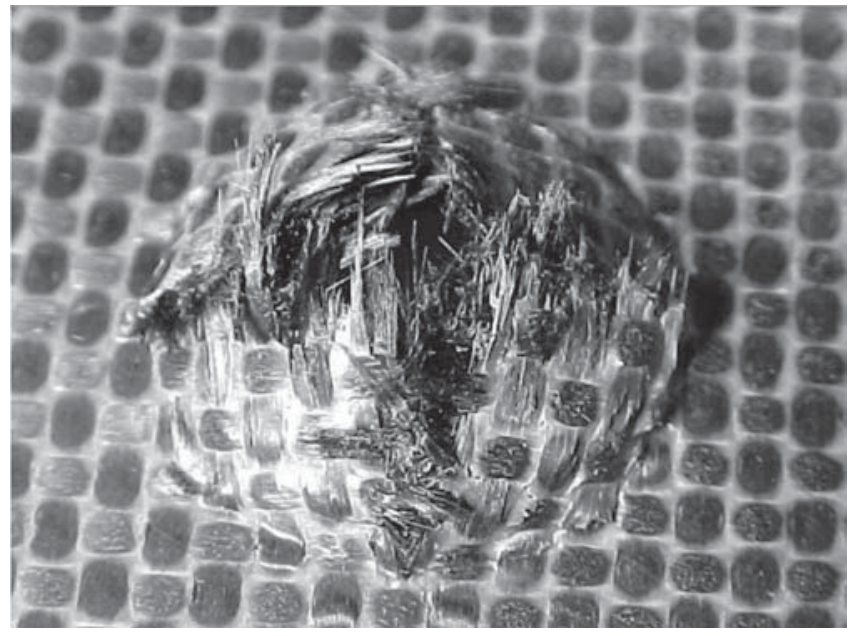

(b) CF-PP/PE (AM2) laminate (opposite side)

Figure 7. Aspects of the CF-PP/PE (AM2) specimens after impact tests with approximately $132 \mathrm{~J}$ of energy.

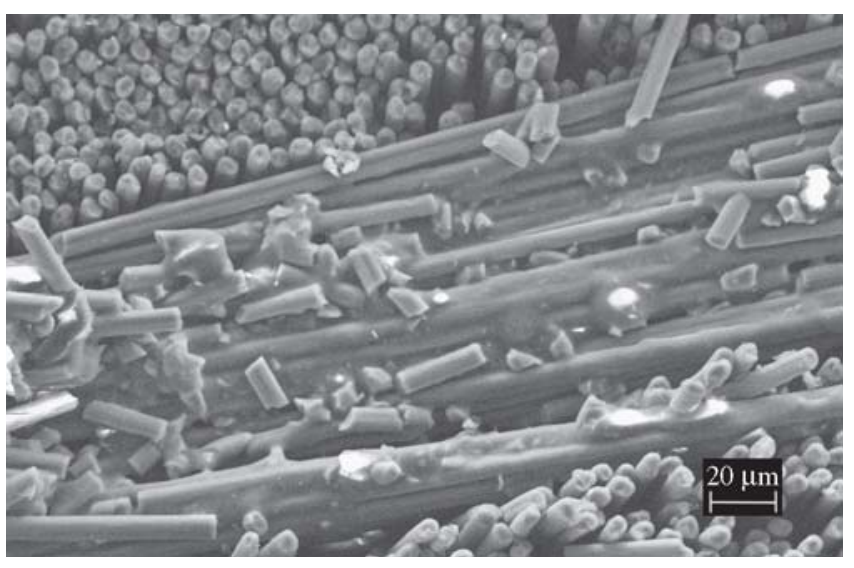

(a) CF-PP laminate fractured $(450 \mathrm{x})$

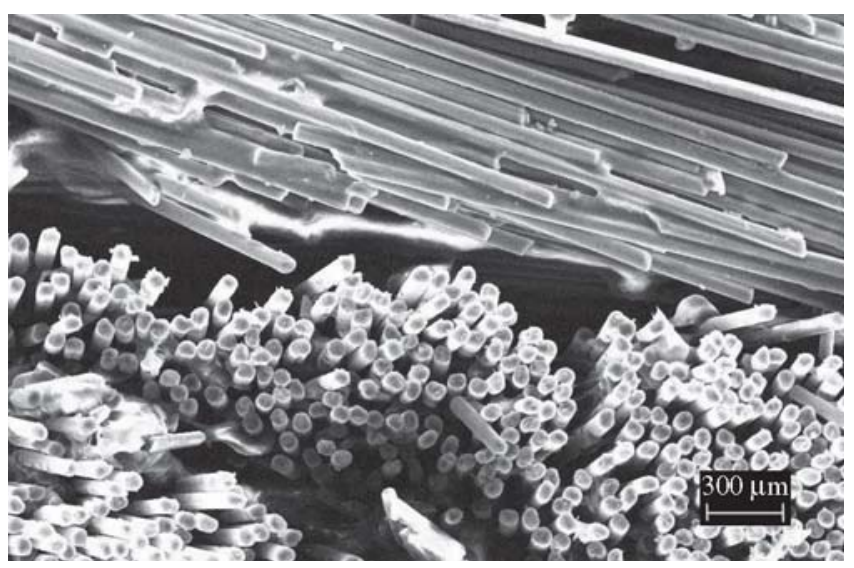

(b) CF-PP/PE laminate fractured $(350 \mathrm{x})$

Figure 8. SEM of fractured specimens after impact tests with approximately $92 \mathrm{~J}$ of energy. 
plastic deformation of the matrix during the tensile test, because of the elastomeric phase (AM2). However, after the tensile tests, the CF-PP/PE-AM2 fractured samples revealed little the effect of plastic deformation of the matrix, prevailing the fiber strength effect.

Figure $10(a-b)$ shows respectively aspects of fractured specimens of the CF-PP and CF-PP/PE laminates after the tensile tests. In this figure it can be observed that the fibers are poorly recovered by the thermoplastic, showing no interfacial adhesion. However, Figure 11 presents several regions containing the thermoplastic matrix recovering the fiber surface, apparently showing better interfacial adhesion between the components. Probably, as discussed for the tensile results, the adhesion is improved by using the AM1 modifier (coupling agent) much better than by using AM2 (elastomeric modifier), in spite of the images not present significant changes.

\section{Conclusions}

According to the scanning electron microscopy observations and the fiber volumetric contents it is verified that the used hot compression molding process (based on a sandwich of carbon fabrics and thermoplastic films) to obtain the thermoplastic laminates is suitable and repetitive. The manufactured laminates showed a homogenous distribution of carbon fibers in the composites, without voids and

Table 5. Tensile strength results of the laminates manufactured.

\begin{tabular}{lccc}
\hline \multicolumn{1}{c}{ Composite } & Tensile Strength (MPa) & \multicolumn{2}{c}{$\begin{array}{c}\text { Modulus of Elasticity } \\
(\mathrm{GPa})\end{array}$} \\
\hline CF-PP & $458.3 \pm 18.6$ & $43.5 \pm 3.1$ & $37.4 \pm 3.4$ \\
CF-PP/PE copolymer & $440.1 \pm 35.9$ & $54.7 \pm 2.4$ & delamination \\
CF-PP/PE (AM1) & $507.6 \pm 11.8$ & $48.6 \pm 5.7$ & fractured \\
CF-PP/PE (AM2) & $422.8 \pm 27.9$ & delamination \\
\hline
\end{tabular}

CF: carbon fiber fabric reinforcement

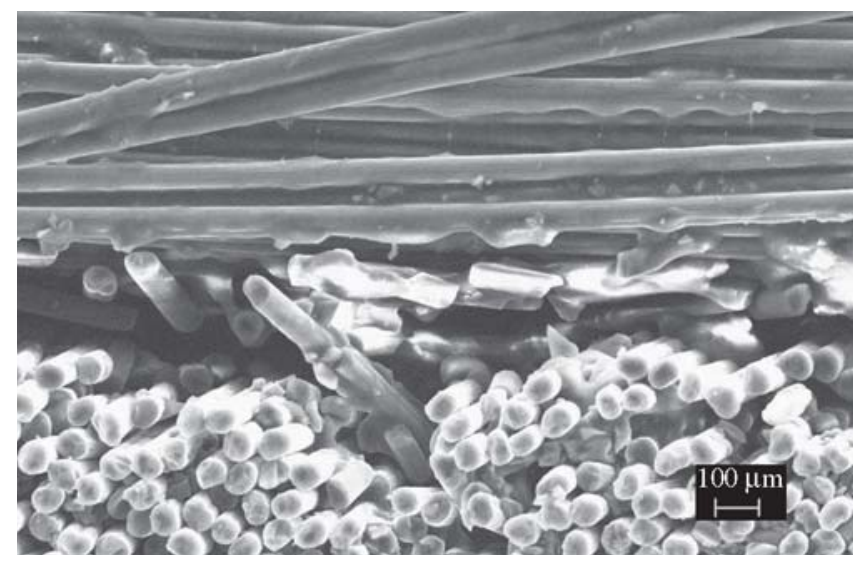

(a) CF-PP (AM1) laminate fractured (600 x)

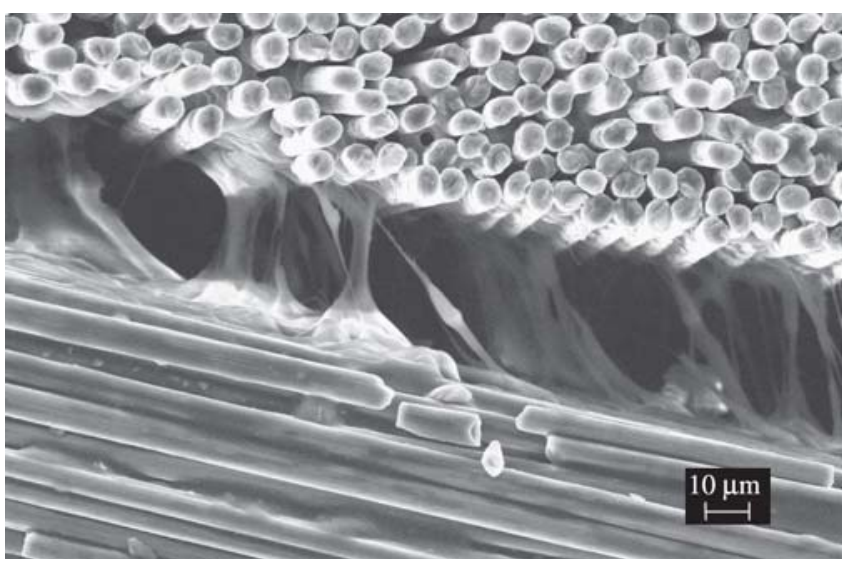

(b) CF-PP/PE (AM2) laminate fractured (600 x)

Figure 9. SEM of fractured specimens after impact tests with approximately $92 \mathrm{~J}$ of energy.

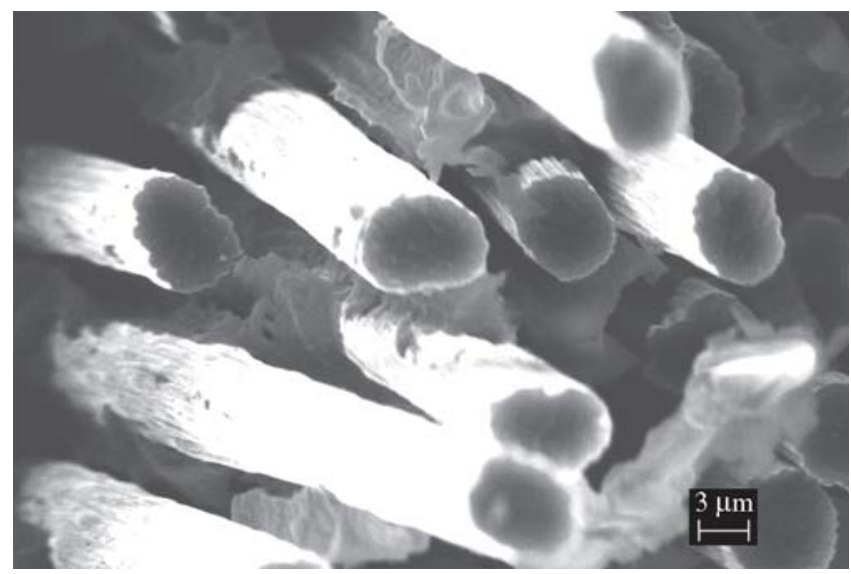

(a) CF-PP laminate $(2000 \mathrm{x})$

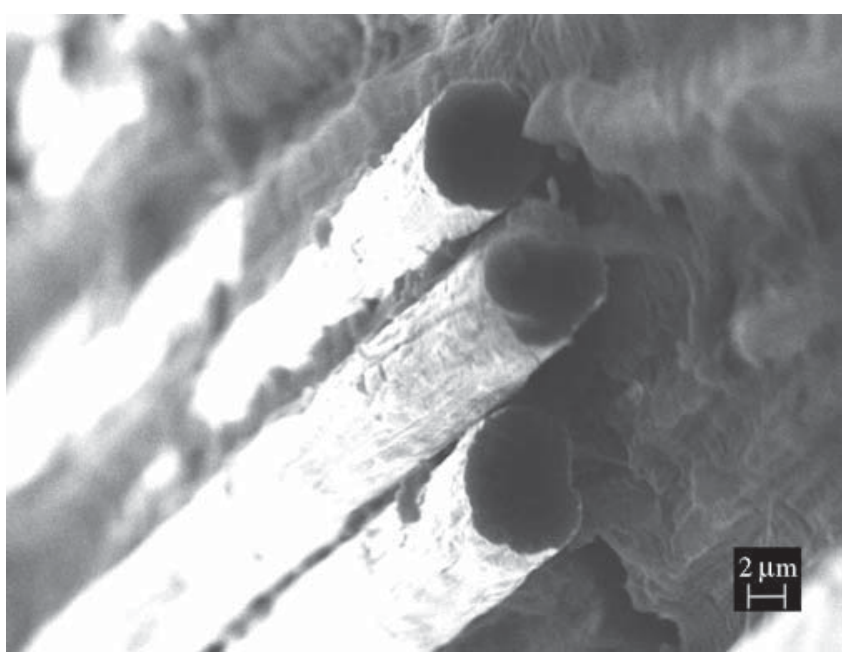

(b) CF-PP/PE laminate $(2000 \mathrm{x})$

Figure 10. SEM of fractured specimens of the CF-PP and CF-PP/PE laminates after tensile tests. 


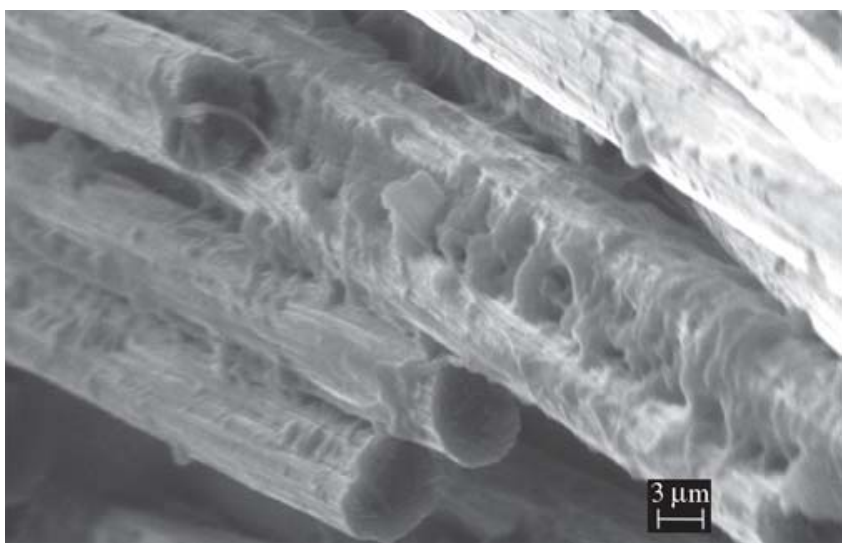

(a) CF-PP/PE-AM1 laminate (2000 x)

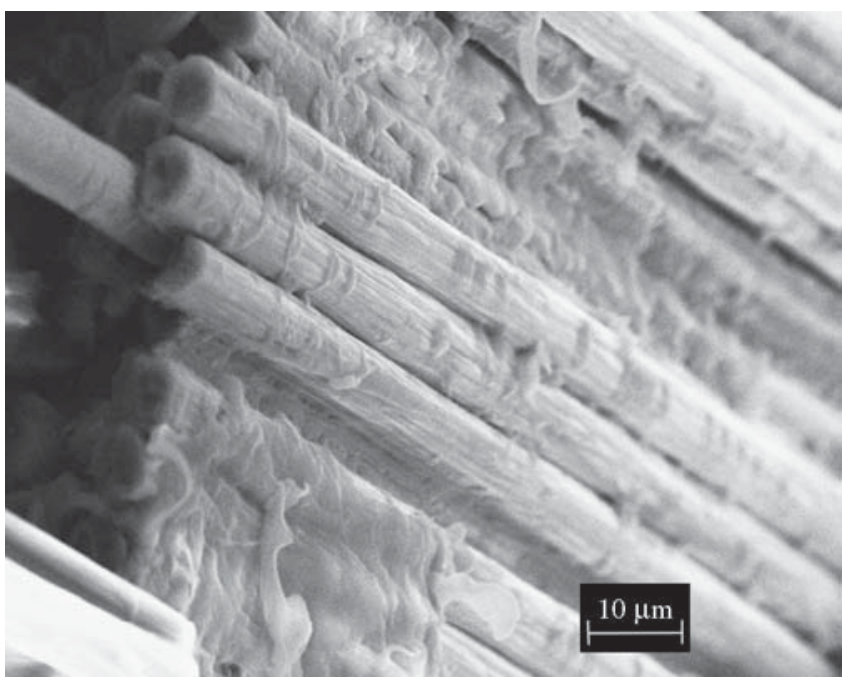

(b) CF-PP/PE-AM2 laminate (2000 x)

Figure 11. SEM of fractured specimens of the CF-PP/PE-AM1 and CF-PP/ PE-AM2 laminates after tensile tests.

cracks and few regions rich in matrix.

The mechanical testing results showed that the PP-PE and PP reinforced laminates presented the lowest impact strength values when compared to the other laminates. However, PP-PE and PP reinforced composite laminates showed a significant increase in the impact strength values when compared respectively to the polymeric matrix plates (without reinforcements).

Correlating the tensile test results and the scanning electron microscopy observations of the tensile fractured surface of the CF-PP/PE-AM1 specimens it is verified a strong interface between fiber/matrix, which leads to the highest tensile strength, but to the lowest impact strength values.

The CF-PP/PE-AM2 laminates presented the lowest tensile strength and the highest impact strength values when compared to the other laminates. Therefore, the CF-PP/PE-AM2 laminate presents the highest capability to resist to impact damages than the other composites because the utilization of AM2 modifier increased the required impact energy to fracture the composite specimens. In this case, the used elastomer called AM2 acted as toughening agent and improved the impact resistance of the composite.

\section{Acknowledgements}

The authors acknowledge the financial support received, respectively, from FAPESP under grant 02/02057-5 and CNPq (303528/2003-6). The authors are indebted to Polibrasil Resinas S/A for supplying the thermoplastic films and to loan the instrumented impact machine.

\section{References}

1. Roder H, Vogl. Progress Polymer Science. 1999; 24:1205-1215.

2. Moore D R, Cervenka, A. Future requirements in the characterization of continuous fiber-reinforced polymeric composites. Pure Applied. Chemistry. 2002; 74(4):601-628.

3. Van De Ven E C, Wijskamp S, Lamers E A D, Akkerman R. Polymer Processing Society, 18, Portugal, 2002.

4. Cabral-Fonseca S, Paiva M C, Nunes J P, Bernardo C A. A novel technique for the interfacial characterisation of glass fibre-polypropylene systems. Polymer Testing. 2003; 22:907-913.

5. Gao S L, Kim J K. Cooling rate influences in carbon fibre/PEEK composites. Composites Part A. 2000; 31:517-530.

6. Smith W. In: Delaware Composites Design Encyclopedia. v.3. USA, Technomic Publishing Co. Inc., p. 15-86, 1990.

7. Schwartz M M. Composite Materials: Properties, Nondestructive testing, and Repair. USA. Prentice Hall PTR, p. 43-52, 1997.

8. Domb M M, Hansen J S. Development of free-edge effect during processing of fibre-reinforced semicrystalline thermoplastic composites. In: Proceedings of 34th SDM Conference, USA, 1994, p. 463-471.

9. Hazen J R, Composites Technology. 2000; 6(3):6-20.

10. Gao S -L, Kim J -K. Cooling rate influences in carbon fibre/PEEK composites. Part III: impact damage performance. Composites, Part A. 2001; 32:775-785.

11. Matthews F L, Rawlings R D. Composite Materials: engineering and science, 2.ed. Boca Raton. Woodhead Publishing Ltd and CRC Press, p. 3-15, 1999.

12. Buchman A, Isayev A I. SAMPE Journal. 1991; 27(4):19-27.

13. Piggott M R. Carbon. 1989; 27(5):657-662.

14. Thanomsilp C, Hogg P J. Penetration impact resistance of hybrid composites based on commingled yarn fabrics. Composites Science and Technology. 2003; 63(3-4):467-482.

15. Dear J P, Brown S A. Impact damage processes in reinforced polymeric materials. Composites Part A. 2003; 34:411-420.

16. Belingardi G, Vadori R. Low velocity impact tests of laminate glassfiber-epoxy matrix composite material plates International. International Journal of Impact and Engineering. 2002; 27(2):213-229.

17. Shyr T -W, Pan Y-H. Impact resistance and damage characteristics of composite laminates. Composite Structures. 2003; 62(2):193-203.

18. Jang B Z. Advanced Polymer Composites: Principles and Applications. ASM International, Materials Park, USA, p. 297, 1994

19. Smith B W. Fractography for continuous fiber composites. In: Engineered Materials Handbook. v.1. USA, Metals Park, OH:ASM, p. 786-793, 1987.

20. Master J E. Basic Failure Modes of Continuous Fiber Composites. In: Engineered Materials Handbook. v. 1. USA, Metals Park, OH:ASM, p. 781-785, 1987. 